Diabetologe $2021 \cdot 17: 516-527$

https://doi.org/10.1007/s11428-021-00785-1

Angenommen: 1. Juni 2021

Online publiziert: 1. Juli 2021

(c) Springer Medizin Verlag GmbH, ein Teil von Springer Nature 2021

In den vergangenen 2 Jahren wurden viele neue Erkenntnisse zu den Themen diabetische Nephropathie und Hypertonie berichtet. Patienten mit diesen Erkrankungen sind besonders häufig unter Menschen mit schweren Verläufen von COVID19 anzutreffen. Eine RAAS-Blockade sollte nicht unterbrochen werden. Für die renale Denervierung gibt es neue Argumente. Die SGLT-2-Inhibitoren mit ihrem günstigen Einfluss bei Nierenfunktionsstörung und Herzinsuffizienz können inzwischen nicht nur bei Typ-2-, sondern auch Typ-1-Diabetes, nichtdiabetischen sowie herzinsuffizienten Patienten eingesetzt werden.

\section{"Coronavirus disease 2019", Diabetes und Hypertonie}

\section{Epidemiologie}

Seit über 1 Jahr ist unser Leben durch die COVID-19-Pandemie geprägt. Schon in den ersten Arbeiten aus China aus dem Jahr 2019 wurde die besondere Häufung von Diabetes, Hypertonie, koronarer Herzerkrankung und Vorhofflimmern bei Menschen mit COVID-19 beschrieben. Publizierte Prävalenzen für den Diabetes lagen bei $12-22 \%$ unter den besonders schweren Verläufen. Das neue

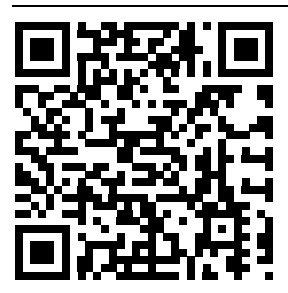

QR-Code scannen \& Beitrag online lesen

Tom H. Lindner

Fachbereich Nephrologie, Klinik und Poliklinik für Endokrinologie, Nephrologie, Rheumatologie (III), Universitätsklinikum Leipzig - AöR, Leipzig, Deutschland

\title{
Neues zu Hypertonie und Diabetes
}

Coronavirus SARS-CoV-2 nutzt ebenso wie sein Vorgänger SARS-CoV-1 den auf bestimmten Geweben exprimierten ACE2-Rezeptor zum Eintritt in die Zellen über die Bindung an das Spikeprotein des Virus. Daher wurde diskutiert, inwieweit der beim Diabetes praktisch standardmäßige Einsatz von RAASBlockern einen Einfluss auf den Krankheitsverlauf von COVID-19 hinsichtlich Schwere und Mortalität ausüben könnte.

In einer Metaanalyse aus 40 Publikationen mit 18.012 Patienten zeigten Almeida-Pittito et al. [1], dass der Diabetes mit einem schwereren Verlauf und einer höheren Mortalität von COVID19 verbunden ist (OR: 2,35 bzw. 2,50); ebenso die Hypertonie (OR: 2,98 bzw. $2,88)$ und kardiovaskuläre Erkrankungen (OR: 4,02 bzw. 6,34). Jedoch beeinflussten weder ACE-Hemmer noch ARB den Krankheitsverlauf.

\section{Kausaler Zusammenhang?}

Die Frage nach dem kausalen Zusammenhang von Diabetes/Hypertonie und dem Verlauf von COVID-19 ist bisher nicht eindeutig geklärt. Huang et al. [2] fanden in einer retrospektiven Studie bei 113 Personen mit Hyper- und 197 mit Normotonie, dass Menschen mit Bluthochdruck häufiger mit SARS-CoV-2 infiziert wurden. Unter Berücksichtigung genereller chinesischer Prävalenzdaten erklärten die Autoren die erhöhte Studienprävalenz der Hypertonie allein über das höhere Alter der Betroffenen. Schweregrad und Mortalität waren nicht vom Vorhandensein einer Hypertonie abhängig.

Iaccarino et al. [3] untersuchten 1521 italienische Patienten mit COVID-19 und fanden eine Hypertonieprävalenz von $54,9 \%$. Hypertonie, Herzinsuffizienz und Geschlecht hatten keinen Einfluss auf den Krankheitsverlauf und die Mortalität. Alter, Diabetes, COPD und CKD waren jedoch assoziiert. Zum Studienzeitpunkt noch aktiv an COVID-19 erkrankt waren $72,7 \%$ der Patienten, sodass die Ergebnisse hochwahrscheinlich kein reales Bild wiedergeben.

Diabetespatienten mit Hypertonie sind höchstwahrscheinlich einem besonderen Risiko für einen schweren Verlauf und eine gesteigerte Mortalität ausgesetzt, wobei die Mechanismen unklar bleiben.

\section{Inhibitoren des Renin- Angiotensin-Aldosteron-Systems und Coronaviruserkrankung}

Rodilla et al. [4] wiesen anhand von Überlebenden und Todesfällen aus 12.226 spanischen Patienten mit COVID-19 einen prädiktiven Einfluss der Hypertonie auf die Mortalität nach, sofern RAAS-Blocker in der Medikation enthalten waren (ACE-Hemmer: OR: 1,3, $p=0,001$; ARB: OR: $1,2, p=0,035)$. Am häufigsten waren auch hier Hypertonie (50,9\%), Diabetes $(19,1 \%)$ und Vorhofflimmern $(11,2 \%)$ anzutreffen. Die Mortalität bei Hypertoniepatienten ohne RAAS-Blockade war höher als im Gesamtstudiendurchschnitt. Das gleiche galt für ACE-Hemmer und ARB, wenngleich die Mortalität hier niedriger als bei den Patienten ohne RAAS-Blockade war.

In den beiden wohl aussagekräftigsten Studien zum Thema RAAS-Blockade unter COVID-19 von Mancia et al. ([5]; Lombardei: 6272 Menschen mit schwe- 


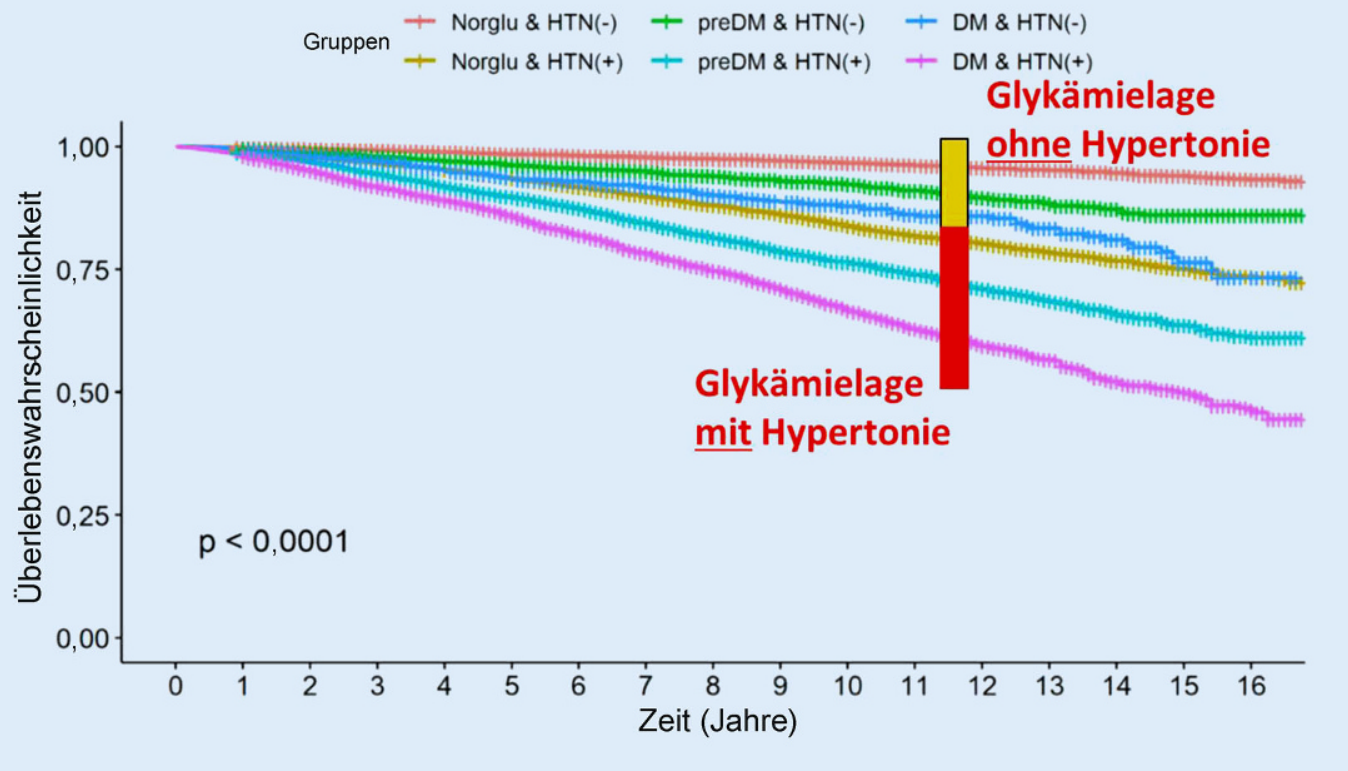

\begin{abstract}
Abb. $1 \varangle$ Kaplan-MeierÜberlebenskurven für Menschen mit Normoglykämie (Norglu), Prädiabetes (preDM) und Diabetes (DM) mit und ohne Hypertonie, HTN(+) mit Hypertonie, HTN(-) ohne Hypertonie. Adaptiert aus [7]
\end{abstract}

rem Verlauf der SARS-CoV-2-Infektion vs. 30.759 Kontrollen, Alter $68 \pm 13$ Jahre, $37 \%$ weiblich) und Reynolds et al. ([6], „New York University“: 12.592 Menschen getestet, 5894 positiv, 1002 schwerer Verlauf; 34,6\% Hypertonie mit 59,1\% positivem Test, $24,6 \%$ mit schwerem Verlauf) wurde keine Beeinflussung des COVID-19-Verlaufs durch ARB oder ACE-Hemmer festgestellt. Weder die Wahrscheinlichkeit der positiven Testung noch die eines schweren Verlaufs waren unter RAAS-Blockade erhöht. Daher sollte eine RAAS-Inhibitor-Therapie unter COVID-19 fortgesetzt werden.

\section{Qualität der Blutdruck- einstellung}

\section{Bedeutung bei Normoglykämie, Prädiabetes und Diabetes}

Um die Frage zu klären, wie sich die Blutdruckeinstellung bei Normoglykämie, Prädiabetes und Diabetes auf die Mortalität auswirkt, wurden Daten aus dem US-amerikanischen NHANESStudienprogramm (40.046 Datensätze, 1999-2014) verwendet [7]. Die niedrigsten kardiovaskulären Mortalitäten ergaben sich für Menschen mit Normoglykämie bei $115-120 \mathrm{~mm} \mathrm{Hg}$, für Prädiabetes bei $120-130 \mathrm{~mm} \mathrm{Hg}$ und für Diabetes bei 125-135 mm Hg. Dabei hatte die Qualität der Blutdruckeinstellung offensichtlich einen deutlich größeren Einfluss als die Diabeteseinstellung: Diabetespatienten ohne Hypertonie hatten zwar ein höheres Risiko als Menschen mit Normoglykämie oder Prädiabetes ohne Hypertonie, aber immer noch ein deutlich niedrigeres Mortalitätsrisiko als Personen mit Normoglykämie/ Prädiabetes/Diabetes und Hypertonie. Bei einem systolischen RR $>150 \mathrm{~mm} \mathrm{Hg}$ war das Sterberisiko von allen untersuchten Gruppen bei Prädiabetes am höchsten ( $\bullet$ Abb. 1). Dies weist darauf hin, dass besonders bei Menschen mit Prädiabetes auf eine gute Blutdruckeinstellung geachtet werden sollte.

\section{Verbesserungspotenzial}

Die Qualität der Blutdruckeinstellung wurde in der KORA-1-Populationsstudie in der Region Augsburg bei 1052 Personen aus der älteren deutschen Bevölkerung (65-94 Jahre) anhand von Prävalenz, bekannter Hypertonie, Blutdruckeinstellung und assoziierten Faktoren untersucht. Die Prävalenz der Hypertonie $(\geq 140 / 90 \mathrm{~mm} \mathrm{Hg})$ lag insgesamt bei $73,8 \%$. Von allen Betroffen wussten $80,2 \%$ von ihrer Hypertonie, und $74,4 \%$ standen unter Antihypertensiva. Diejenigen, die von ihrer Hypertonie wussten, waren $\mathrm{zu} 92,8 \%$ therapiert, davon befanden sich 53,7\% im Zielbereich. Bluthochdruck trat erwartungs- gemäß bei den Älteren, Adipösen oder Diabetespatienten häufiger auf.

Mit diesen Daten erreicht Deutschland international einen Spitzenplatz. Dennoch bleibt bei 53,7\% Zielerreichung ein großes Verbesserungspotenzial. Bestandteile der antihypertensiven Therapie wie strikte Kombinationstherapie, standardisierte Adhärenztestungen im Urin sowie die besondere Betonung der Verbesserung des Lebensstils, insbesondere einer adäquaten Ernährung und vermehrten körperlichen Aktivität von Beginn an, müssen als wesentliche Bestandteile der Therapie noch viel mehr in den Vordergrund gerückt werden.

\section{Bedeutung des mittleren arteriellen Blutdrucks}

Eine $\mathrm{zu}$ niedrige Blutdruckeinstellung kann insbesondere bei Älteren die Organdurchblutung kritisch herabsetzen und das Gegenteil des Gewünschten bewirken. Blutdruck und Blutfluss werden in ihrer pulsatilen Form als systolischer und diastolischer Blutdruck bestimmt, wobei es sich um die Extremwerte der Schwingung um den mittleren arteriellen Druck handelt, der die Organperfusion bestimmt. Wenn der periphere Widerstand steigt, nimmt der MAP zusammen mit dem systolischen und dem diastolischen Druck zu. Wenn sich jedoch die größeren Gefäße versteifen, steigen zwar 
systolischer Blutdruck und MAP, aber der diastolische Blutdruck fällt.

Melgarejo et al. [8] schlugen vor, bei der ambulanten Blutdruckmessung über $24 \mathrm{~h}$ den MAP als Orientierungshilfe für die Therapie mit anzeigen zu lassen. Sie ermittelten anhand von Daten von 11.596 Patienten die MAP-Bereiche, die zu den Schwellenwerten der in den Leitlinien festgelegten SBP- und DBP-Werte passten und gleichzeitig mit der gleichen Zahl kardiovaskulärer Ereignisse einhergingen. Das 10-JahresRisiko für den primären kombinierten Endpunkt (kardiovaskuläre Mortalität einschließlich plötzlicher Herztod, nichttödliche koronare Ereignisse; koronare Revaskularisation, Herzinsuffizienz und Apoplex) war konsistent höher mit steigendem MAP bei einem zusätzlichen Einfluss des systolischen Blutdrucks, wohingegen höhere diastolische Werte das Risiko abschwächten. Der MAP sollte also sowohl bei der Identifizierung eines Bluthochdrucks als auch in der Therapieeinstellung berücksichtigt werden. Das kardiovaskuläre Risiko wird mit niedriger eingestelltem systolischem Blutdruck sinken, aber nur, wenn der MAP und der diastolische Blutdruck nicht $\mathrm{zu}$ einer kritischen Organperfusion führen (• Tab. 1).

In einer Metaanalyse [9] wurde der blutdrucksenkende Effekt von Liraglutid bei 968 Typ-2-Diabetes-Patienten aus 10 RCT mit einer Beobachtungszeit von $16 \pm 9$ Wochen untersucht. Liraglutid in einer Konzentration von 1,8 mg/Tag senkte den SBP um 5,39 $\mathrm{mm} \mathrm{Hg}$ und das Gewicht um 2,07 kg. Die Verminderungen von SBP- und Gewicht standen bei den mit Liraglutid behandelten Diabetespatienten nicht im Zusammenhang.

Marre [10] fasste den aktuellen Wissenstand zur Hypertoniediagnostik und -therapie insbesondere für Diabetespatienten zusammen (u.a. - Tab. 2).

\section{Renale Denervierung - wieder im Fokus}

Generell kommt die interventionelle Blutdrucksenkung zur Anwendung, wenn medikamentöse Therapien versagen. Es handelt sich sozusagen um die letzte Möglichkeit, um einer zu-

Diabetologe 2021 · 17:516-527 https://doi.org/10.1007/s11428-021-00785-1

(c) Springer Medizin Verlag GmbH, ein Teil von Springer Nature 2021

\section{T. H. Lindner}

\section{Neues zu Hypertonie und Diabetes}

\section{Zusammenfassung}

Hypertonie und Diabetes treten oft gemeinsam auf und erhöhen die kardiovaskuläre Mortalität. Beide Erkrankungen sind bei COVID-19-Patienten (COVID-19: „coronavirus disease 2019“) mit schweren Verläufen gehäuft anzutreffen. Hinsichtlich der Kausalität für COVID-19 ist die Datenlage widersprüchlich. Sicher ist ein Surrogateffekt, da meiste ältere COVID-19-Patienten einen schweren Verlauf aufgrund der Vorschäden aufweisen. Hemmer des Renin-AngiotensinAldosteron-Systems (RAAS-Blocker) erhöhen nicht die Wahrscheinlichkeit der positiven SARS-CoV-2-Testung (SARS-CoV-2: „severe acute respiratory syndrome coronavirus $2^{\prime \prime}$ ). Sie haben keinen Einfluss auf die Schwere des Verlaufs und die Mortalität und sollten daher unter COVID-19 nicht pausiert/abgesetzt werden. Die renale Denervierung erfreut sich einer Renaissance, nachdem sich die Studiendesigns und Technologien stark weiterentwickelt haben. Bei den SGLT-2Hemmern (SGLT-2: „sodium glucose linked transporter 2") ist der Wissenszuwachs enorm.
In den ersten beiden großen randomisierten kontrollierten Studien mit primären renalen Endpunkten verzögerten sie die Progression der chronischen Niereninsuffizienz (CKD) bis zum Dialysebeginn deutlich um zusätzliche ca. 12-13 Jahre. Sie sind prinzipiell bei Typ-2- sowie Typ-1-Diabetes und auch bei herzinsuffizienten Patienten mit und ohne Diabetes einsetzbar und reduzieren den systolischen Blutdruck. Der nichtsteroidale Mineralokortikoidrezeptorantagonist (MRA) Finerenon wirkt bei Typ-2-Diabetes reno- und kardioprotektiv bezüglich kardiovaskulärer Mortalität und Niereninsuffizienz. Wie bei den klassischen MRA kann es auch hier zur Hyperkaliämie in höheren CKD-Bereichen kommen. Größere Vergleichsstudien zu den klassischen MRA liegen nicht vor. Die Zulassung in Deutschland steht noch aus.

Schlüsselwörter

Diabetische Nephropathie - Bluthochdruck . COVID-19 · SGLT-2-Inhibitoren · Renale Denervation

\section{Recent trends in hypertension and diabetes therapy}

\section{Abstract}

Hypertension and diabetes often appear together and increase cardiovascular mortality. Both diseases do frequently occur in patients with a severe course of COVID19 (coronavirus disease 2019). Data in the literature are contradictory. A surrogate effect seems to be likely since older patients do often present a severe course due to their pre-existing pathologic conditions. Renin-angiotensin-aldosterone system (RAAS) inhibitors do not increase the probability of a positive test for SARS-CoV-2 (severe acute respiratory syndrome coronavirus 2 ). They have no impact on the severity or mortality and must not be discontinued. Renal denervation reappears after study designs and technologies developed much further. Knowledge regarding SGLT2 (sodium glucose linked transporter 2) inhibitors has been increased enormously. The first two large randomized controlled trials with primary renal endpoints emerged and demonstrated a significantly slower progression of diabetic nephropathy postponing the initialization of chronic hemodialysis therapy by about 12 to 13 years. SGLT2 inhibitors can be used in type 2 and type 1 diabetic patients and patients with heart failure with or without diabetes. They decrease the systolic blood pressure independent of the basic blood pressure. However, this effect does not explain the improvement of cardiorenal endpoints. The nonsteroidal mineralocorticoid receptor antagonist (MRA) finerenone is protective for heart and kidneys in type 2 diabetic patients regarding cardiovascular mortality and kidney failure. As with classic MRAs hyperkalemia is seen with this substance as well, particularly in higher chronic kidney disease stages. Larger studies comparing classic MRAs do not exist. Finerenone approval in Germany is pending.

\section{Keywords}

Diabetic nephropathy - Blood pressure, high · COVID-19 · SGLT2 inhibitors · Renal denervation 
Tab. 1 Stadieneinteilung der WHO an-

hand systolischer, diastolischer und mittlerer arterieller Drücke

\begin{tabular}{|c|c|c|}
\hline $\begin{array}{l}\text { 24-h-MAP } \\
\text { [mm Hg] }\end{array}$ & WHO & $\begin{array}{l}\text { SBP/DBP } \\
{[\mathrm{mm} \mathrm{Hg}]}\end{array}$ \\
\hline$<90$ (normal) & Normal & $<130 / 85$ \\
\hline $90-<92$ & $\begin{array}{l}\text { Hoch- } \\
\text { normal }\end{array}$ & $130-139 / 85-89$ \\
\hline $92-<96$ & WHO-1 & 140-159/90-99 \\
\hline$\geq 96$ & WHO-2 & 160-179/100-109 \\
\hline \multicolumn{3}{|c|}{$\begin{array}{l}\text { DBP diastolischer Blutdruck („diastolic blood } \\
\text { pressure“), MAP mittlerer arterieller Blutdruck } \\
\text { („,mean arterial pressure”), SBP systolischer } \\
\text { Blutdruck („,systolic blood pressure“), WHO Welt- } \\
\text { gesundheitsorganisation („World Health Organi- } \\
\text { zation") }\end{array}$} \\
\hline
\end{tabular}

künftigen kardiovaskulären Problematik vorzubeugen.

Die letzte Dekade war von einem Auf und $\mathrm{Ab}$ bezüglich der RDN als Mittel zur Blutdrucksenkung geprägt: zunächst von Euphorie, da in den ersten Studien von unterschiedlicher Größe, Design und Methoden signifikante Blutdrucksenkungen erzielt wurden (u.a. SYMPLICITY HTN-1/-2). Dann folgten die Dämpfung bis Desillusionierung durch die Ergebnisse der Studien ReSET und SYMPLICITY HTN-3: In diesen randomisierten, verblindeten, scheinprozedurkontrollierten Untersuchungen fand sich kein Unterschied mehr zwischen den Behandlungsgruppen. In Posthoc-Analysen wurden die Heterogenität der Patienten sowie verschiedene Katheter- und Behandlungsprotokolle als Ursachen für die fehlenden blutdrucksenkenden Effekte verantwortlich gemacht.

In den Studien der zweiten Generation (RADIANCE-HTN SOLO, SPYRAL HTN-OFF MED, SPYRAL HTN-ON MED) wurden Probanden mit einer isolierten systolischen Hypertonie ausgeschlossen und hochqualifiziertes Personal zur Durchführung der RDN hinzugezogen. Multipolare Katheter, erweiterte Ablation der Seitenäste, Verwendung von ultraschallbasierter RDN, modifizierte therapeutische Regimes und der Einsatz der ambulanten Blutdruckmessung wurden inkorporiert, und die blutdrucksenkenden Effekte stellten sich wieder ein.
Jüngst analysierten Mahfoud et al. [11] Daten aus dem globalen SYMPLICITYRegister mit der Fragestellung, ob das kardiovaskuläre Basisrisiko die Effizienz der RDN bei Patienten mit hohem kardiovaskulärem Risiko über 3 Jahre nach RDN beeinflusst. In der Gesamtkohorte $(n=2652)$ sank der SBP nach 3 Jahren um $-8,9 \pm 20,1 \mathrm{~mm}$ Hg. Die Blutdrucksenkungen zu den Zeitpunkten 6, 12, 24 und 36 Monate verhielten sich ähnlich und konsistent über alle Subgruppen und die verschiedenen Risikoscores für arteriosklerotische kardiovaskuläre Erkrankungen hinweg $(p<0,0001 ; \bullet$ Tab. 3 , - Abb. 2). Der Blutdruckabfall hing nicht vom basalen kardiovaskulären Risiko ab, obwohl sich, wie erwartet, mehr kardiovaskuläre Ereignisse in den höheren Risikogruppen zeigten. Hier müssen die

Hier steht eine Anzeige.

\section{量 Springer}

Resultate größerer Studien mit einer Scheinprozedurkontrollgruppe und mit kardiovaskulären Endpunkten abgewartet werden.

Mahfoud et al. [12] führten zudem mit dem Peregrine System ${ }^{\mathrm{mm}}$ Infusion Catheter eine neuartige Technologie ein, bei welcher dehydrierter Alkohol als neurolytisches Agens, das in den periarteriellen Raum der Nierenarterien injiziert wird, verwendet wurde. In einer multizentrischen, Open-Label-Studie konnten die Effizienz und Sicherheit dieser Technik nachgewiesen werden. Der primäre Sicherheitsendpunkt (Nichtauftreten von periprozeduralen großen Gefäßkomplikationen, großen Blutungen, AKI oder Tod innerhalb eines Monats) wurde bei $96 \%$ der Patienten erfüllt. Jedoch gab es auch hier keine Scheinprozedurgruppe.

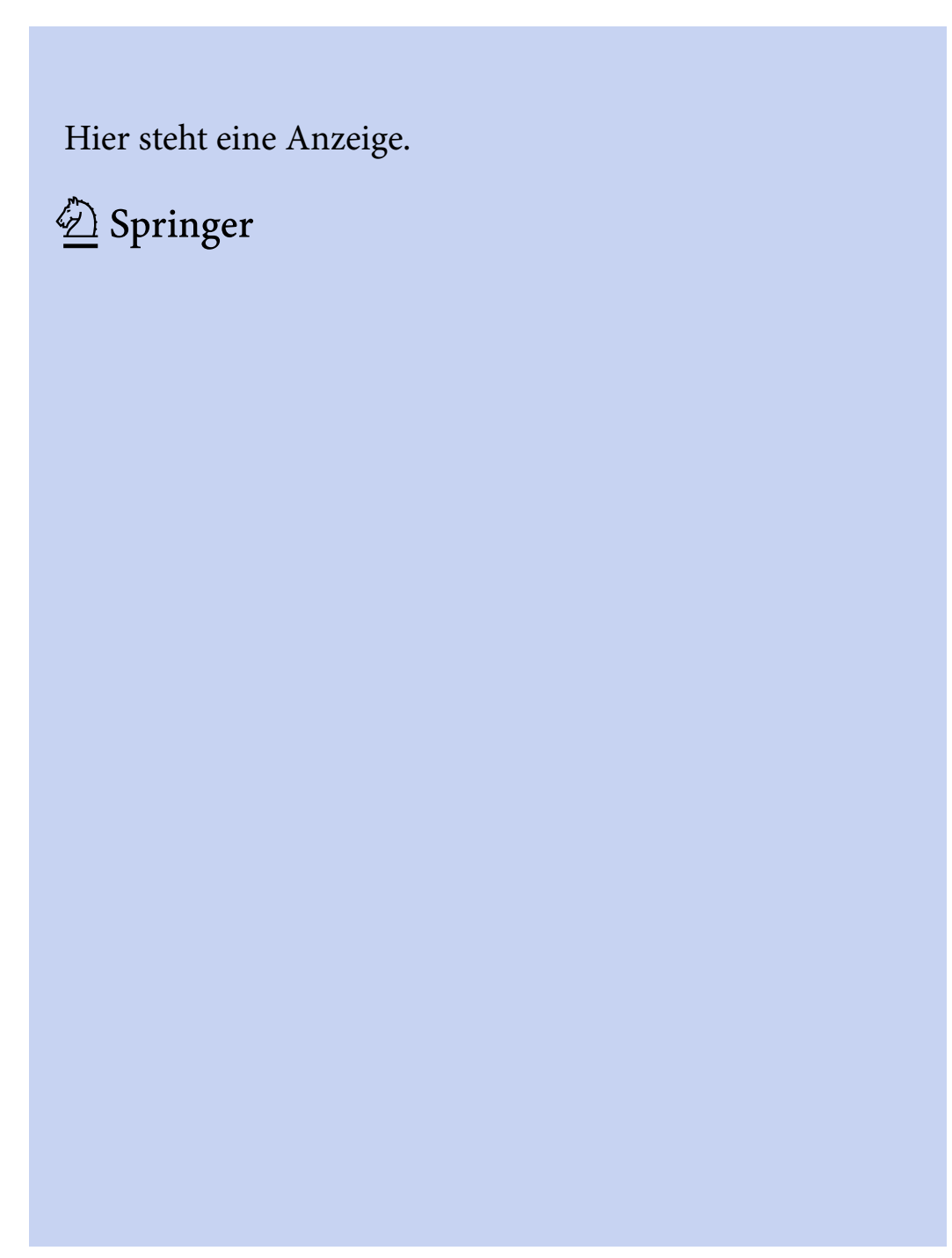


Tab. 2 Aktueller Stand der Therapie mit klassischen Antihypertensiva und antihypertensiv wirkenden Antidiabetika

Klasse Gruppe Aktueller Anwendungsstand

Klassische An- RAAS-Inhibi- Therapie der ersten Wahl

tihypertensiva toren

Duale Blockade wird nicht empfohlen

Diuretika Werden eingesetzt, ohne dass spezifische Outcomestudien für Schleifendiuretika, Thiazide und Thiazidanaloga existieren

Kalzium- Die meisten Studien wurden mit Dihydropyridinen durchgeführt, die ohne Kombination mit RAAS-Blocker eine Protekanalblocker inurie sogar verschlimmern können. In Kombination wird jedoch die antiproteinurische Wirkung verstärkt

Betablocker Bevorzugte Medikamentengruppe bei KHK und Herzinsuffizienz

Die Kombination mit einem Thiazid ist einer Kombinationstherapie aus RAAS-Inhibitor + Kalziumkanalblocker klar unterlegen. Deswegen gehören Betablocker außerhalb der KHK/Herzinsuffizienz nicht zu den vorrangig eingesetzten Medikamenten. Nichtselektive Betablocker verschlechtern die Glukosetoleranz. Der selektive Betablocker Nebivolol weist diese Problematik nicht auf. Für die meisten neueren selektiven Betablocker gibt es jedoch keine guten Daten. Am häufigsten wurde Atenolol eingesetzt

a-Blocker, Für Diabetespatienten gibt es dazu keinerlei Outcomestudien

Nitroprussid,

Minoxidil

Antidiabetika Sulfonyl-

mit potenziell harnstoffe

antihyperten-

siver Wirkung
Grundsätzlich inhibieren die SH die Wirkung der CCB-Therapie. In der UKPD-Studie unterschieden sich die Patienten mit und ohne SH hinsichtlich RR-Einstellung jedoch nicht. In der ADVANCE-Studie senkte der Einsatz von Gliclazid den systolischen Blutdruck um $2 \mathrm{~mm} \mathrm{Hg}$, ohne dass dieses Phänomen bis heute erklärt werden kann. Daher werden insgesamt die Sulfonylharnstoffe gegenüber dem Blutdruck und dem kardiovaskulären Risiko als neutral betrachtet

Metformin Bei moderat adipösen Patienten in der UKPDS senkte Metformin das Herzinfarktrisiko um $39 \%$, ohne allerdings Auswirkungen auf den Blutdruck zu zeigen

DPP-4-In- Saxagliptin, Alogliptin, Sitagliptin und Linagliptin zeigten keine signifikanten Wirkungen auf den Blutdruck. Sie werhibitoren den als kardiovaskulär neutral betrachtet

GLP-1-RA In Phase-2-/-3-Studien reduzierten sie den systolischen Blutdruck und erhöhten die Herzfrequenz (ähnlich wie Vasodilatatoren und Diuretika). Die Auswirkung auf das RAAS ist unklar. Liraglutid scheint Angiotensin-ll etwas zu senken, was auch den anderweitig gezeigten leichten Blutdruckabfall unter GLP-1-RA erklären könnte

SGLT-2-In- In bisherigen CV-Outcome-Studien sank der RR um 5-7 mm Hg

hibitoren

CCB Kalziumkanalblocker ("calcium channel blocker"), CV kardiovaskulär, DPP-4 Dipeptidylpeptidase 4, GLP-1-RA Rezeptoragonisten des „glucagon-like peptide 1", KHK koronare Herzkrankheit, RAAS Renin-Angiotensin-Aldosteron-System, RR Blutdruck nach Riva-Rocci, SGLT-2 "sodium glucose linked transporter 2", SH Sulfonylharnstoffe

Tab. 3 Blutdrucksenkung durch renale

Denervierung nach 3 Jahren

\section{Subgruppe SBP-Senkung}

nach 3 Jahren

Resistente Hypertonie $\quad-10,4 \pm 21,0 \mathrm{~mm} \mathrm{Hg}$

Patienten $\geq 65$ Jahre $\quad-8,7 \pm 17,4 \mathrm{~mm} \mathrm{Hg}$

Diabetes $\quad-10,2 \pm 17,9 \mathrm{~mm} \mathrm{Hg}$

Isolierte systolische $\quad-8,6 \pm 18,7 \mathrm{~mm} \mathrm{Hg}$

Hypertonie

CKD $\quad-10,1 \pm 20,3 \mathrm{~mm} \mathrm{Hg}$

Vorhofflimmern $\quad-10,0 \pm 19,1 \mathrm{~mm} \mathrm{Hg}$

CKD chronische Niereninsuffizienz (I, chronic

kidney disease"), SBP systolischer Blutdruck

(,systolic blood pressure")

\section{Hemmer des Natrium-Glukose- Kotransports}

\section{Antihypertensive Wirkung}

Die EMPA-REG OUTCOME ${ }^{\circledR}$-Studie zeigte 2015 bei 7020 Typ-2-DiabetesPatienten unter Empagliflozin eine Senkung der kardiovaskulären Mortalität (primärer Endpunkt) und eine Verbesserung sekundärer renaler Endpunkte. Seitdem wurde diese Studie unter verschiedenen Fragestellungen post-hoc weiter analysiert. All diese Folgebetrachtungen beruhten auf nichtvorspezifizierten Subgruppen, sodass die Ergebnisse allenfalls als hypothesengenerierend und mit nur geringer Evidenz angesehen werden sollten.

Ferreira et al. [13] identifizierten 1579 Patienten mit einer angenommenen resistenten Hypertonie ( $\geq 3$ Antihypertensiva mit nicht ausreichend eingestelltem $\mathrm{RR} \geq 140 / \geq 90 \mathrm{~mm} \mathrm{Hg}$ oder $\geq 4$ Antihypertensiva [einschließlich Diuretikum] mit eingestelltem RR), die über 3,1 Jahre nachbeobachtet worden waren. Die mittlere Differenz des systolischen Blutdrucks zwischen Studienbeginn und Woche 12 lag für prHT vs. Plazebo bei $-4,5 \mathrm{~mm} \mathrm{Hg}(p<0,001)$ und für $\mathrm{Pa}$ tienten ohne prHT bei $-3,7 \mathrm{mmHg}$ $(p<0,001)$. Unter Empagliflozin erreich- ten mehr Betroffene den Zielblutdruck $(<130 / 80 \mathrm{~mm} \mathrm{Hg})$. Patienten mit einer angenommenen resistenten Hypertonie hatten im Vergleich zu Menschen ohne prHT ein 1,5- bis 2-fach erhöhtes Risiko einer Hospitalisierung wegen Herzinsuffizienz, inzidenter/sich verschlechternder Nephropathie und von kardiovaskulärem Tod. Empagliflozin verbesserte alle Outcomeparameter sowohl bei Patienten mit als auch ohne prHT.

\begin{tabular}{l}
\hline Empagliflozin verbesserte alle \\
Outcomeparameter unabhängig \\
von einer evtl. vorhandenen \\
Hypertonie
\end{tabular}

Böhm et al. [14] zeigten anhand der EMPA-REG OUTCOME ${ }^{\circledR}$-Daten, dass Empagliflozin im Vergleich zu Plazebo den systolischen Blutdruck bei 


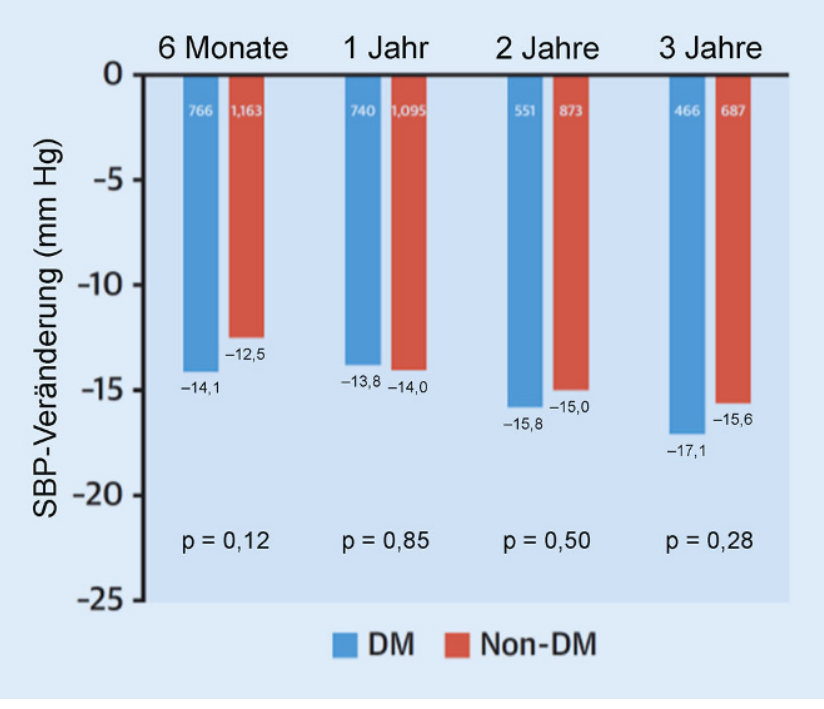

Abb. $2<$ Veränderung des SBP (systolischer Blutdruck [, systolic blood pressure"]) für Diabetes- (DM) und Nichtdiabetespatienten (Non $D M)$ über 3 Jahre. Adaptiert aus [11]
Diabetespatienten mit und ohne Herzinsuffizienz unabhängig vom AusgangsSBP senkte. Die signifikante Verbesserung kardiovaskulärer und renaler Endpunkte einschließlich der Verbesserung der Mortalität wurde nicht durch die SBPSenkung bewirkt. Nur für die inzidente oder sich verschlechternde Nephropathie zeigte sich eine mediierende Wirkung des SBP.

Jung et al. [15] prüften in einer prospektiven Arbeit bei 97 Typ-2-Diabetes-Patienten die vaskuläre Wirkung verschiedener Kombinationstherapien. Zunächst wurden die Probanden mit Metformin mindestens 3 Monate vorbehandelt. Danach erhielten sie über die Randomisierung entweder Empagliflozin, $10 \mathrm{mg} / \mathrm{Tag}$, und Linagliptin; $5 \mathrm{mg} / \mathrm{Tag}(\mathrm{E}+\mathrm{L})$, oder sie erhielten weiterhin Metformin, 2-mal 850 oder $1000 \mathrm{mg} /$ Tag plus nun zusätzlich Insulin Glargin $(\mathrm{M}+\mathrm{I})$. Nach 12 Wochen $\mathrm{E}+\mathrm{L}$ sanken Praxisdruck und 24-hBlutdruck, wohingegen unter M + I keine Änderungen zu beobachten waren. Demnach wäre die Kombination Empagliflozin + Linagliptin der Kombination Metformin + Insulin für diese spezifische Situation vorzuziehen. Inzucchi et al. [16] beobachteten, dass Empagliflozin als add-on zu Metformin bei Typ-2Diabetes-Patienten über einen Verlauf von 76 Wochen den $\mathrm{HbA}_{1 \mathrm{c}}$, das Körpergewicht und den SBP gegenüber Plazebo senkte. Ausgangswerte $>140 \mathrm{~mm} \mathrm{Hg}$ wurden signifikant um 5,42 $\mathrm{mm} \mathrm{Hg}$ und
Ausgangswerte $<130 \mathrm{mmHg}$ nur tendenziell um 3,68 mm Hg gesenkt.

Auch Dapagliflozin wirkt bei Typ-2Diabetes blutdrucksenkend [17, 18]. Insgesamt ist diese Wirkung der SGLT-2Hemmung nicht auf die gleichzeitige Gewichtssenkung zurückführbar.

\section{Nephroprotektive Wirkung der Natrium-Glukose-Kotransport- Inhibitoren}

\section{Post-hoc-Analysen von EMPA- REG OUTCOME ${ }^{\circledR}$}

Nachdem die US-amerikanische FDA 2016 unter Canagliflozin vermehrt Fälle von akutem Nierenversagen festgestellt hatte, wurde dieser Gesichtspunkt in mehreren Studien aufgearbeitet. Gilbert et al. [19] und Neuen et al. [20] fanden in ihren Metaanalysen, die EMPAREG OUTCOME ${ }^{\circledR}$, DECLARE-TIMI58 und CANVAS einschlossen, sogar weniger Fälle von akutem Nierenversagen.

In weiteren Post-hoc-Analysen der EMPA-REG OUTCOME ${ }^{\circledR}$-Studie wurden die sekundären renalen Endpunkte mit anderen Fragestellungen beleuchtet:

- Nach Levin et al. [21] verbesserte

Empagliflozin über alle untersuch-

ten eGFR-Klassen hinweg (60-90, $>30-<60,15-30,<15 \mathrm{ml} / \mathrm{min}$ ) die Nierenfunktion.

- Kadowaki et al. [22] beschrieben für Asiaten die gleiche Verbesserung der CKD-Progression durch Empagliflozin wie in der gesam- ten Studienpopulation von EMPAREG OUTCOME $^{\circledR}$.

- Bei Diabetespatienten mit und ohne eine bekannte und angegebene Herzinsuffizienz bewirkte Empagliflozin gleichermaßen eine verzögerte Progression der diabetischen Nephropathie [23].

- Teilnehmer der EMPA-REG OUTCOME $^{\circledR}$-Studie standen unter verschiedenen antihyperglykämischen Therapien, darunter auch einigen, die das kardiovaskuläre Risikoprofil möglicherweise beeinflussen könnten. Von 7020 Diabetespatienten erhielten $74 \%$ Metformin, $43 \%$ Sulfonylharnstoffpräparate und $48 \%$ Insulin zum Studienbeginn (allein oder in Kombination). Die Risikoreduktionen für 3-PunktMACE, Hospitalisierung wegen Herzinsuffizienz und Gesamtmortalität waren in allen Subgruppen der Basistherapien konsistent nachweisbar. Unter Metformin fiel jedoch die Risikoreduktion geringer aus als bei Menschen, die diesen Wirkstoff nicht einnahmen [24].

- In der EMPA-REG OUTCOME ${ }^{\circledR}$ Studie sank die eGFR initial um 3-5 $\mathrm{ml} / \mathrm{min}$ (sog. eGFR-Dip), was hochwahrscheinlich mit intrarenalen hämodynamischen Effekten zusammenhängt. Kraus et al. [25] teilten 6668 Teilnehmer mit verfügbaren eGFR-Werten zum Zeitpunkt 0 und Woche 4 anhand ihrer eGFRVeränderungen über die beiden Zeitpunkte in 3 Gruppen ein: $>10 \%$ Absenkung, >0-10\% Absenkung, $0 \%$ Absenkung. Ein initialer eGFRDip wurde in $28,3 \%$ der mit Empagliflozin behandelten gegenüber 13,4\% der Plazebo erhaltenden Teilnehmer beobachtet (OR: 2,7). In der multivariaten logistischen Regression waren Diuretika und eine höhere KDIGO-Risikokategorie unabhängig voneinander prädiktiv für den eGFR-Dip unter Empagliflozin vs. Plazebo. Allerdings hatte der eGFRDip keinen wesentlichen Einfluss auf die Endpunkte kardiovaskulärer Tod, Hospitalisierung wegen 


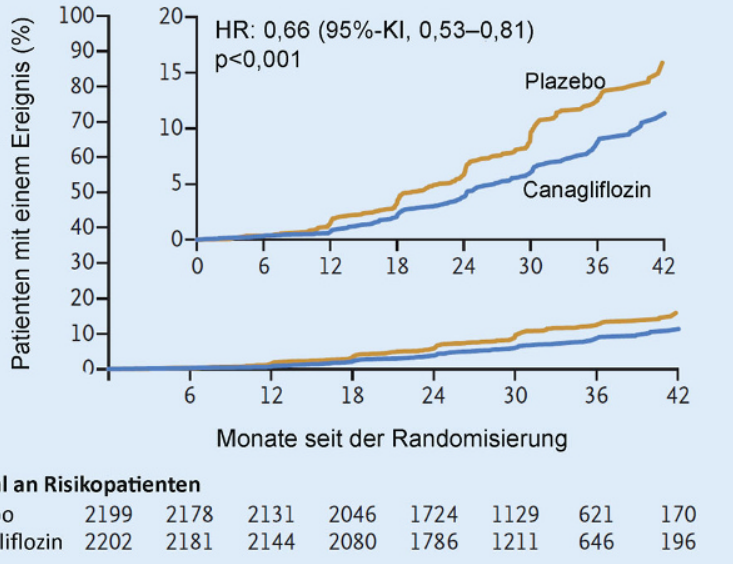

Herzinsuffizienz und inzidente/sich verschlechternde Nephropathie [25].

\section{Empagliflozin und sekundäre renale Endpunkte bei Herzinsuffizienz}

In die EMPA-REG OUTCOME ${ }^{\circledR}$-Studie wurden nur Typ-2-Diabetes-Patienten mit und ohne eine bekannte und angegebene Herzinsuffizienz aufgenommen. Im Jahr 2020 wurde die EMPEROR-Reduced-Studie publiziert, die ausschließlich herzinsuffiziente Patienten mit NYHAStadien II, III oder IV mit einer Ejektionsfraktion $\leq 40 \%$ mit und ohne Typ-2Diabetes einschloss. Die Probanden wurden neben der Standardtherapie entweder mit Empagliflozin $10 \mathrm{mg} / \mathrm{Tag}$ oder Plazebo behandelt [26]. Der primäre Endpunkt war eine Kombination von kardiovaskulärem Tod oder Hospitalisierung wegen sich verschlechternder Herzinsuffizienz. Die renale Betrachtung erfolgte lediglich über sekundäre Endpunkte. Nach einem Median von 16 Monaten trat der primäre Endpunkt in der Empagliflozingruppe bei 361 von 1863 Patienten $(19,4 \%)$ und in der Plazebogruppe bei 462 von 1867 Patienten $(24,7 \%)$ auf (HR: 0,75, $p<0,001)$. Der Effekt von Empagliflozin war bei Patienten mit und ohne Typ-2-Diabetes gleichermaßen nachweisbar. Die Absolutzahl der Hospitalisierungen wegen Herzinsuffizienz war der wesentliche Faktor und lag in der Empagliflozingruppe niedriger als in der Plazebogruppe (HR: 0,70; $p<0,001)$. Auch der jährliche eGFRVerfall war in der Empagliflozingrup- pe niedriger als in der Plazebogruppe ( $-0,55$ vs. $-2,28 \mathrm{ml} / \mathrm{min} / \mathrm{Jahr}, p<0,001)$. Außerdem zeigten die Patienten unter Empagliflozin ein niedrigeres Risiko für renale Endpunkte.

\section{Canagliflozin - erste Studie eines Natrium-Glukose-Kotransporters mit primären renalen Endpunkten}

Perkovic et al. [27] veröffentlichten 2019 die CREDENCE-Studie, in der mit Canagliflozin erstmalig für die SGLT-2-Inhibitoren primär renale Endpunkte untersucht wurden. Dabei wurden 4401 Typ-2-Diabetes-Patienten auf $100 \mathrm{mg} / \mathrm{Tag}$ Canagliflozin oder Plazebo randomisiert (eGFR $30-<90 \mathrm{ml} / \mathrm{min}$, UACR $>300-5000 \mathrm{mg} / \mathrm{g}$ Kreatinin im Urin, 99,9\% unter RAAS-Blockade). Der primäre Endpunkt war eine Kombination von terminaler Niereninsuffizienz (Dialyse, Transplantation oder permanente eGFR $<15 \mathrm{ml} / \mathrm{min}$ ), Verdopplung des Serumkreatinins oder Tod durch Herz-/ Nierenversagen. Die Studie wurde wegen eindeutigem Vorteil von Canagliflozin hinsichtlich Mortalität nach 2,62 Jahren vorzeitig abgebrochen. Das relative Risiko für den primären Endpunkt lag unter Canagliflozin 30\% niedriger als unter Plazebo mit Ereignisraten von 43,2 vs. $61,2 / 1000$ Patientenjahre (HR: $0,70, p=0,00001)$. Das relative Risiko der nierenspezifischen Kombination aus terminalem Nierenversagen, Verdopplung des Serumkreatinins oder Tod durch Nierenversagen lag unter Canagliflozin um $34 \%$ niedriger (HR: $0,66, p<0,001$; - Abb. 3). Das Risiko des terminalen

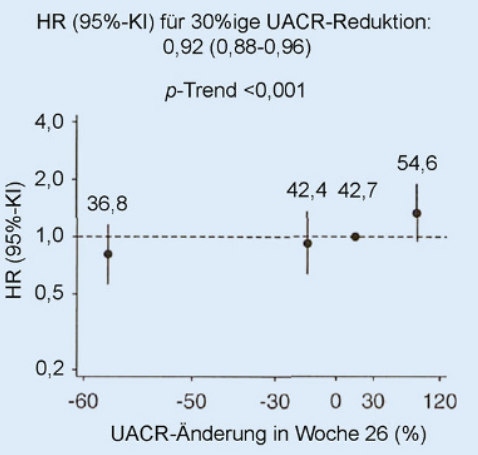

Abb. 4 \ $H R$ (,hazard ratio") bezüglich schwerer kardiovaskulärer Komplikationen in Bezug auf die UACR-Änderung nach 26 Wochen unter Canagliflozin, 95\%-KI 95\%-Konfidenzintervall, UA$C R$ Urin-Albumin-Kreatinin-Verhältnis („urinealbumin-creatinine ratio"). Adaptiert aus [29]

Nierenversagens allein war unter Canagliflozin $32 \%$ geringer als unter Plazebo (HR: $0,68, p=0,002$ ). Die Effekte waren über den gesamten untersuchten eGFRBereich zwischen 30 und $90 \mathrm{ml} / \mathrm{min}$ nachweisbar (Interaktions- $p$ n. s.). In der Canagliflozingruppe traten auch weniger kardiovaskuläre Todesfälle, Myokardinfarkte oder Schlaganfälle (HR: 0,80, $p=0,01)$ sowie weniger Hospitalisierungen wegen Herzinsuffizienz (HR: $0,61, p<0,001)$ auf. Insgesamt reduzierte Canagliflozin eindeutig die Mortalität und verlangsamte die Progression der Nephropathie.

In einer weiteren Post-hoc-Analyse berechneten Durkin et al. [28], dass sich durch die Behandlung mit Canagliflozin die Dialysepflichtigkeit um 12,95 Jahre hinausschieben lässt - ein beeindruckendes Ergebnis! Oshima et al. [29] wiederum wiesen in einer Post-hocAnalyse der CREDENCE-Studie nach, dass besonders der frühzeitige Rückgang der Albuminurie in den ersten Monaten der Behandlung mit Canagliflozin im Langzeitverlauf mit signifikant weniger MACE, insbesondere weniger kardiovaskulären Todesfällen und weniger Hospitalisierungen wegen Herzinsuffizienz, assoziiert war (• Abb.4).

Kopfzerbrechen bereitet die initiale eGFR-Reduktion in der Plazebogruppe. Denn nicht nur $45 \%$ der Patienten unter Canagliflozin, sondern auch $21 \%$ der Probanden aus der Plazebogruppe 
wiesen einen initialen eGFR-Abfall von $>10 \%$ (signifikante OR: 3,03) auf. Bei $4 \%$ der Patienten unter Canagliflozin und $2 \%$ derjenigen unter Plazebo betrug dieser sogar $>30 \%$. Der Abfall der eGFR im weiteren Verlauf verlief in allen eGFR-Kategorien gleichermaßen.

\section{》) Unter Canagliflozin verbes- sern sich die Nierenparameter}

Auf den klinischen Befund hatte die initiale Abnahme der eGFR keinen Einfluss. Ein akuter Abfall derselben in der Canagliflozingruppe wurde eher bei älteren $\mathrm{Pa}$ tienten mit längerer Diabetesdauer, höherem BMI, höherem systolischem Blutdruck, höherer eGFR, geringerer Wahrscheinlichkeit einer Herzinsuffizienz und vermehrt unter Diuretikatherapie beobachtet. In der Plazebogruppe waren höherer systolischer Blutdruck, höhere eGFR und stärkere UACR für einen initialen eGFR-Abfall prädiktiv, was möglicherweise auf ein Problem in der Therapieadhärenz vor Beginn der Interventionsstudie zurückzuführen sein könnte. Laut der Publikation standen 99,9\% der Patienten unter einer RAAS-Blockade. Diese führt, genauso wie SGLT-2-Inhibitoren, zu einem initialen Abfall der eGFR. Die anfängliche Reduktion der eGFR in der Plazebogruppe könnte somit durchaus durch eine bessere Adhärenz zu den zuvor eingenommenen RAAS-Blockern zustande gekommen sein.

Auch Patienten mit einer eGFR $<30 \mathrm{ml} / \mathrm{min}$ profitieren (möglicherweise). Bei 174 Probanden der CREDENCEStudie lag die eGFR zum Zeitpunkt des Screenings bei $\geq 30 \mathrm{ml} / \mathrm{min}$, zum Zeitpunkt der Randomisierung jedoch bei $<30 \mathrm{ml} / \mathrm{min}$, weswegen sie von der Hauptstudie ausgeschlossen wurden. Diese Klientel wurde in einer Post-hocAnalyse noch einmal gesondert betrachtet [30]. Von Wochen 1-130 imponierte eine 66\%ige Differenz des mittleren eGFR-Abfalls mit Canagliflozin vs. Plazebo ( $-1,30$ vs. $-3,83 \mathrm{ml} / \mathrm{min} / \mathrm{Jahr}$; Differenz-2,54 [0,90-4,17] ml/min/Jahr). Die Effekte auf die renalen und kardiovaskulären Endpunkte waren für die eGFR < 30 und $\geq 30 \mathrm{ml} / \mathrm{min}$ konsistent. In beiden eGFR-Gruppen gab es keine wesentli- chen Unterschiede hinsichtlich renaler Nebenwirkungen oder akuter Niereninsuffizienz. Interessanterweise zeigte sich für den eGFR-Bereich $<30 \mathrm{ml} / \mathrm{min}$ kein initialer Abfall. Dies weist darauf hin, dass hier die Hyperfiltration keine Rolle mehr spielt und dennoch die eGFR im Verlauf von der Medikation profitiert. Jedoch ist bei diesen Ergebnissen Vorsicht angezeigt: Die Dynamik einer chronischen Niereninsuffizienz in dieser Klientel ist möglicherweise nicht repräsentativ für die gesamte Stichprobe abgebildet. Die meisten der Patienten lagen mit ihrer eGFR bei $20-30 \mathrm{ml} / \mathrm{min}$. Valide Rückschlüsse für den eGFR-Bereich $<30 \mathrm{ml} / \mathrm{min}$ können daher noch nicht abgeleitet werden.

\section{DAPA-CKD-Studie - Effekte von Dapagliflozin bei Diabetes- und Nichtdiabetespatienten}

Als zweite RCT mit primär renalen Endpunkten nach CREDENCE folgte die DAPA-CKD-Studie ([33]; Typ-2Diabetes mit CKD: $n=4302$; eGFR $=25-75 \mathrm{ml} / \mathrm{min}, \mathrm{UACR}=200-5000 \mathrm{mg} / \mathrm{g}$ Kreatinin im Urin, unter $10 \mathrm{mg} / \mathrm{Tag} \mathrm{Da}-$ pagliflozin vs. Plazebo). Der primäre Endpunkt war eine Kombination aus persistierendem Abfall der eGFR um $\geq 50 \%$, ESRD oder kardiovaskulärem Tod/Tod durch Nierenversagen.

In die CREDENCE-Studie wurden nur Typ-2-Diabetes-Patienten eingeschlossen. Hingegen wiesen in der DAPA-CKD-Studie (Phase 3) 67\% der Probanden einen Typ-2-Diabetes auf, was eine zusätzliche Aussage für Nichtdiabetespatienten erlaubte (allerdings beruhte die Diagnose Diabetes lediglich auf $\mathrm{HbA}_{1 \mathrm{c}}$-Werten, der Aktenlage und nicht auf Nüchternblutglukosebestimmungen oder oGTT). Wie zuvor die CREDENCE-Studie wurde auch DAPACKD wegen eindeutiger Überlegenheit von Dapagliflozin nach 2,4 Jahren vorzeitig abgebrochen. Der primäre Endpunkt wurde von 197 der 2152 Teilnehmer (9,2\%) aus der Dapagliflozingruppe und von 312 der 2152 Probanden (14,5\%) der Plazebogruppe erreicht (HR: 0,61 [95\%KI: 0,51-0,72], $p<0,001$; NNT, um ein Ereignis $\mathrm{zu}$ verhindern: 19 [95\%-KI: 15-27]). Die HR für die Kombination aus persistierender eGFR-Absenkung $\geq 50 \%$, ESRD oder kardiovaskulärem Tod/Tod durch Nierenversagen lag bei $0,56(p<0,001)$. Die HR für die Kombination aus kardiovaskulärem Tod oder Hospitalisierung wegen Herzinsuffizienz war 0,71 $(p=0,009)$. In der Dapagliflozingruppe gab es 101 Todesfälle $(4,7 \%)$ vs. 146 (6,8\%) in der Plazebogruppe (HR: $0,69, p=0,004)$. Die Dapagliflozineffekte unterschieden sich nicht zwischen Diabetes- und Nichtdiabetespatienten [31].

Nach der DAPA-CKD-Studie folgte eine Reihe von hochinteressanten Posthoc-Analysen:

- In der getrennten Post-hoc-Betrachtung von Diabetes- und Nichtdiabetespatienten zeigten Wheeler et al. [32], dass beide Gruppen gleichermaßen von Dapagliflozin hinsichtlich nierenspezifischem Outcome, kardiovaskulärem Tod oder Hospitalisierungen wegen Herzinsuffizienz und der Gesamtmortalität profitierten.

- Entsprechend den Phase-3-Studien DEPICT-1 (11/2014-08/2017) und DEPICT-2 (07/2015-04/2018) verbesserte Dapagliflozin zusätzlich zu Insulin bei einem schlecht eingestellten Typ-1-Diabetes die Stoffwechselkontrolle und das Körpergewicht, ohne das Risiko einer Hypoglykämie zu steigern. Nun konnten Groop et al. [33] zusätzlich anhand der Daten der DEPICT-Studien nachweisen, dass Dapagliflozin im Vergleich zu Plazebo die Albuminausscheidung im Urin über 52 Wochen senkte $(5 \mathrm{mg} /$ Tag: $-13,3 \%$; $10 \mathrm{mg} /$ Tag: $-31,1 \%)$. Dabei kam es zu keiner signifikanten Veränderung der eGFR ( $5 \mathrm{mg} /$ Tag: $3,27 \mathrm{ml} / \mathrm{min}$; $10 \mathrm{mg} /$ Tag: $2,12 \mathrm{ml} / \mathrm{min}$; [33]).

- In der DAPASALT-Studie ([34], nichtrandomisiert, „open label“) ging es um die zugrunde liegenden Mechanismen der geringeren Mortalität unter SGLT-2-Inhibitoren. Der Fokus lag auf Natriumausscheidung und osmotischer Diurese. Hierfür wurden 14 Typ-2-DiabetesPatienten unter Dapagliflozin auf eine kontrollierte standardisierte Salzdiät gesetzt (150 mmol/Tag). Evaluiert wurden Natriumausscheidung, ambulante 24-h-Blutdruckwerte, 


\begin{tabular}{|c|c|c|c|}
\hline \multicolumn{2}{|c|}{ Abkürzungen } & \multicolumn{2}{|c|}{ Abkürzungen (Fortsetzung) } \\
\hline \multirow[t]{2}{*}{ 24-h-ABPM } & \multirow{2}{*}{$\begin{array}{l}\text { Ambulante Messung des Blut- } \\
\text { drucks („ambulatory blood pres- } \\
\text { sure measurement“) über } 24 \mathrm{~h}\end{array}$} & $R R$ & Blutdruck nach Riva-Rocci \\
\hline & & \multirow[t]{2}{*}{ SARS-COV } & \multirow{2}{*}{$\begin{array}{l}\text { "Severe acute respiratory } \\
\text { syndrome coronavirus" }\end{array}$} \\
\hline$A C E$ & Angiotensinkonversionsenzym & & \\
\hline$A K I$ & $\begin{array}{l}\text { Akute Nierenschädigung („acute } \\
\text { kidney injury“) }\end{array}$ & $S B P$ & $\begin{array}{l}\text { Systolischer Blutdruck („systolic } \\
\text { blood pressure“) }\end{array}$ \\
\hline$A R B$ & Angiotensinrezeptorblocker & SGLT-2 & $\begin{array}{l}\text { "Sodium glucose linked transpor- } \\
\text { ter } 2 "\end{array}$ \\
\hline$B M I$ & Body-Mass-Index & \multirow[t]{2}{*}{ UACR } & \multirow{2}{*}{$\begin{array}{l}\text { Urin-Albumin-Kreatinin-Verhält- } \\
\text { nis („,urine-albumin-creatinine } \\
\text { ratio“) }\end{array}$} \\
\hline$C K D$ & $\begin{array}{l}\text { Chronische Niereninsuffizienz } \\
\text { („chronic kidney disease“) }\end{array}$ & & \\
\hline
\end{tabular}

COPD Chronisch obstruktive Lungenerkrankung (",chronic obstructive pulmonary disease")

\begin{tabular}{|c|c|c|}
\hline COVID-19 & „Coronavirus disease 2019“ & am Ende (Tage 12-14) und an den \\
\hline$D B P$ & $\begin{array}{l}\text { Diastolischer Blutdruck („diastolic } \\
\text { blood pressure“) }\end{array}$ & $\begin{array}{l}\text { Tagen } 15-18 \text { (Follow-up). Über die } \\
\text { gesamte Behandlungsphase verän- }\end{array}$ \\
\hline DSP & „Digital systolic blood pressure" & ng \\
\hline eGFR & $\begin{array}{l}\text { Geschätzte glomeruläre Filtrati- } \\
\text { onsrate („estimated glomerular } \\
\left.\text { filtration rate }{ }^{\prime \prime}\right)\end{array}$ & $\begin{array}{l}\text { signifikant von } 128 \mathrm{~mm} \mathrm{Hg} \mathrm{um} \\
6,1 \mathrm{~mm} \mathrm{Hg}(p<0,001) \text { nach } 2-4\end{array}$ \\
\hline ESRD & „End-stage renal disease“ & 12-14 Tagen (Beendigung der The- \\
\hline$F D A$ & $\begin{array}{l}\text { "U.S. Food and Drug Administra- } \\
\text { tion" }\end{array}$ & $\begin{array}{l}\text { rapie) ab. Dapagliflozin veränderte } \\
\text { nicht das Plasma- oder das Intra- }\end{array}$ \\
\hline$H b A_{1 c}$ & Glykiertes Hämoglobin Typ $A_{1 c}$ & \\
\hline$H R$ & „Hazard ratio" & $2-4$ Tagen um 0,71 abfiel $(p=0,02)$. \\
\hline KDIGO & $\begin{array}{l}\text { „Kidney disease: improving } \\
\text { global outcomes" }\end{array}$ & $\begin{array}{l}\text { Diuretische Effekte können nach } \\
\text { dieser, wenn auch kleinen, Studie die }\end{array}$ \\
\hline MACE & $\begin{array}{l}\text { Schwere kardiale Komplikation } \\
\text { („major adverse cardiac event“) }\end{array}$ & $\begin{array}{l}\text { Mortalitätssenkung nicht erklären. } \\
\text { Vieles spricht inzwischen dafür, dass }\end{array}$ \\
\hline MAP & $\begin{array}{l}\text { Mittlerer arterieller Blutdruck } \\
\text { („mean arterial pressure“) }\end{array}$ & $\begin{array}{l}\text { dieser Benefit auf eine bessere Ver- } \\
\text { fügbarkeit von } \beta \text {-Hydroxy-Butyrat }\end{array}$ \\
\hline MRA & Mineralokortikoidrezeptor- & zurückzuführen ist. \\
\hline
\end{tabular}

NHANES "National health and nutrition examination survey"

\begin{tabular}{|ll}
\hline NNT & „Number needed to treat" \\
\hline NYHA & "New York Heart Association“ \\
\hline oGTT & Oraler Glukosetoleranztest \\
\hline OR & "Odds ratio“ \\
\hline prHT & $\begin{array}{l}\text { Angenommen resistente } \\
\text { Hypertonie („presumed resistant } \\
\text { hypertension“) }\end{array}$ \\
\hline$R A A S$ & $\begin{array}{l}\text { Renin-Angiotensin-Aldosteron- } \\
\text { System }\end{array}$ \\
\hline$R C T$ & $\begin{array}{l}\text { Randomisierte kontrollierte } \\
\text { Studie („randomized controlled } \\
\text { trial“) }\end{array}$ \\
\hline$R D N$ & \begin{tabular}{l} 
Renale Denervierung \\
\hline
\end{tabular} \\
\hline
\end{tabular}

kaliämie, Gynäkomastie) einher, die ihren Einsatz limitieren.

In derFIDELIO-DKD-Studie (Phase 3, [35]) wurden bei 5734 Typ-2 DiabetesPatienten mit CKD die renalen und kardiovaskulären Langzeitwirkungen einer Therapie mit dem nichtsteroidalen MRA Finerenon untersucht. Die Studienteilnehmer wurden 1:1 auf Finerenon $(n=2833)$ oder Plazebo $(n=2841)$ randomisiert und im Median über 2,6 Jahre beobachtet (Einschlusskriterien: UACR $30-<300 \mathrm{mg} / \mathrm{g}$ Kreatinin im Urin, eGFR $25-<60 \mathrm{ml} / \mathrm{min}$ mit diabetischer Retinopathie oder UACR 300-5000 mg/g Kreatinin im Urin mit einer eGFR $25-<75 \mathrm{ml} / \mathrm{min})$. Alle Patienten standen bereits zu Studienbeginn unter maximal möglicher/tolerierbarer RAASBlockade. Der primäre Endpunkt war eine Kombination aus Nierenversagen (ESRD oder Nierentransplantation oder eGFR $<15 \mathrm{ml} / \mathrm{min}$ ), eine persistierende eGFR-Absenkung von $\geq 40 \%$ im Vergleich zum Ausgangspunkt oder Tod durch Nierenversagen.

Der primäre Endpunkt trat bei 504 $(17,8 \%)$ Patienten der Finerenongruppe und $600(21,1 \%)$ Probanden der Plazebogruppe auf (HR: 0,82, $p=0,001$ ). Der sekundäre Endpunkt war eine Kombination aus kardiovaskulärem Tod, nichttödlichem Herzinfarkt, nichttödlichem Schlaganfall oder Hospitalisierung wegen Herzinsuffizienz und trat bei 367 (13,0\%) Patienten der Finerenongruppe und $420(14,8 \%)$ Teilnehmern der Plazebogruppe auf (HR: $0,86, p=0,03$ ). Die Rate der Nebenwirkungen war in beiden Gruppen ungefähr gleich. Ein hyperkaliämiebedingter Abbruch wurde häufiger unter Finerenon $(2,3 \%)$ als unter Plazebo beobachtet $(0,9 \%)$. Typ-2-DiabetesPatienten mit CKD zeigten unter Finerenon im Vergleich zu Plazebo ein geringeres Risiko für ein Fortschreiten der CKD. Auch die Zahl der sekundären kardiovaskulären Endpunkte nahm unter Finerenon ab. Eine eGFR $<45 \mathrm{ml} / \mathrm{min}$ hatten $55 \%$ der Patienten. Trotz fortgeschrittener CKD profitierten sie von der Finerenontherapie: Die renalen Finerenoneffekte wurden nach 12 Monaten signifikant, die sekundären kardiovaskulären Endpunkte bereits nach 1 Monat. Die zugehörigen Kurven der Plazebo- 
Hier steht eine Anzeige.

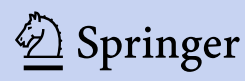


bzw. Finerenongruppe wichen jedoch schon viel früher voneinander ab. Dies weist auf akute Stoffwechseleffekte durch Finerenon hin. Als solche akute Wirkung kommt die Suppression des sekundären Hyperaldosteronismus bezüglich des kardiovaskulären Geschehens in Frage. Dies bessert ebenfalls die Albuminausscheidung im Urin, da Aldosteron üblicherweise proteinurisch wirkt. In der Diskussion führten die Autoren allerdings eher antiinflammatorische und antifibrotische Wirkungen an, die für MRA bekannt sind und die späten Wirkungen nach 12 Monaten erklären könnten.

Die beschriebenen Effekte zeigten sich in der Vergangenheit auch unter den klassischen Aldosteronantagonisten Spironolacton und Eplerenon, sodass bei bisher praktisch fehlenden Vergleichen (nur sehr kleine Sicherheitsarbeiten mit zu guter eGFR) eine Überlegenheit von Finerenon noch nachzuweisen ist. Dennoch lassen sich gewisse Schlussfolgerungen ziehen:

- Unter der nichtsteroidalen Substanz

Finerenon kommt es ebenso zur Hyperkaliämie wie unter klassischen MRA. In der Finerenongruppe kamen Hyperkaliämien bei 446 (15,8\%) der Patienten vor, vs. 221 (7,8\%) in der Plazebogruppe. Die Autoren argumentierten diesbezüglich, dass der Abbruch wegen Hyperkaliämie unter Finerenon auf RAAS-Inhibitoren wesentlich seltener auftrat als in den Studien zur üblichen dualen RAAS-Blockade (2,3\% vs. 4,8\% [Aliskiren + ACE-Inhibitoren/ARB] bzw. 9,2\% [ACE-Inhibitoren + ARB]).

- Das ähnliche Verhalten bezüglich Hyperkaliämie lässt vermuten, dass Finerenon gegenüber Spironolacton oder Eplerenon keine Vorteile bringt. In die FIDELIO-DKD-Studie wurden Typ-2-Diabetes-Patienten mit fortgeschrittener CKD eingeschlossen, bei denen eine Hyperkaliämie aufgrund der schlechten Nierenfunktion in der Regel häufiger auftritt. In der Vergangenheit wurden bei den Sicherheitsstudien für Finerenon eher Patienten mit $z u$ guter eGFR und damit nur marginalem Risiko einer Hyperkaliämie betrachtet. Dennoch wurde die hier scheinbar fehlende Hyperkaliämie vorzeitig als Argument pro Finerenon genutzt.

- Der zweite potenzielle Vorteil könnte die Verhinderung der Gynäkomastie durch einen nunmehr nichtsteroidalen MRA sein. In der vorliegenden Studie wurde leider nicht bezüglich dieser Nebenwirkung untersucht.

Finerenon wirkt entsprechend den Daten der FIDELIO-DKD-Studie kardiound nephroprotektiv. Ein Vergleich mit den klassischen MRA existiert bis auf sehr kleine Studien mit nur milder CKD nicht. Aussagen zur Unter- oder Überlegenheit oder Gleichwertigkeit können derzeit noch nicht getroffen werden.

\section{Fazit für die Praxis}

- „Coronavirus disease 2019“ (COVID-19) tritt gehäuft bei Hypertonie und Diabetes auf. Inhibitoren des Renin-Angiotensin-AldosteronSystems (RAAS) haben keinen Einfluss auf die Infektion, die Schwere des Verlaufs und die Mortalität und sollten daher bei COVID-19 nicht abgesetzt werden.

- Inhibitoren von SGLT-2 („,sodium glucose linked transporter $2^{\prime \prime}$ ) können eingesetzt werden bei Typ-2Diabetes (alle), Typ-1-Diabetes (Sotagliflozin, Dapagliflozin), Herzinsuffizienz mit und ohne Typ-2-Diabetes (Dapagliflozin)

- SGLT2-Hemmer senken den systolische Blutdruck unabhängig vom Ausgangsblutdruck und der gesenkten Mortalität, bei Patienten mit/ ohne Herzinsuffizienz.

- Inhibitoren von SGLT-2 verlangsamen die Progression der diabetischen Nephropathie (etwa 12 Jahre Verzögerung der Dialysepflichtigkeit).

- Finerenon als nichtsteroidaler Mineralokortikoidrezeptorantagonist (MRA) wirkt bei Typ-2-Diabetes renound kardioprotektiv. Größere vergleichende Studien zu den klassischen MRA stehen jedoch noch aus.

\section{Korrespondenzadresse}

Prof. Dr. med. Tom H. Lindner

Fachbereich Nephrologie, Klinik und Poliklinik für Endokrinologie, Nephrologie, Rheumatologie (III), Universitätsklinikum Leipzig - AöR

Liebigstraße 20, 04103 Leipzig, Deutschland tom.lindner@medizin.uni-leipzig.de

\section{Einhaltung ethischer Richtlinien}

Interessenkonflikt. T.H. Lindner gibt Vortragstätigkeit bei Novo Nordisk, AstraZeneca, Sanofi, Amgen, Berlin-Chemie und Beratertätigkeit bei Vifor, Boehringer-Ingelheim an.

Für diesen Beitrag wurden vom Autor keine Studien an Menschen oder Tieren durchgeführt. Für die aufgeführten Studien gelten die jeweils dort angegebenen ethischen Richtlinien.

\section{Literatur}

1. de Almeida-Pititto B, Dualib PM, Zajdenverg L, Dantas JR, de Souza FD, Rodacki M, Bertoluci MC (2020) Severity and mortality of COVID 19 in patients with diabetes, hypertension and cardiovascular disease: a meta-analysis. Diabetol Metab Syndr 12:75. https://doi.org/10.1186/ s13098-020-00586-4

2. Huang S, Wang J, Liu F, Liu J, Cao G, Yang C et al (2020) COVID-19 patients with hypertension have more severe disease: a multicenter retrospective observational study. Hypertens Res 43:824-831. https://doi.org/10.1038/s41440-020-0485-2

3. laccarino G, Grassi G, Borghi C, Ferri C, Salvetti M, Volpe M (2020) Age and multimorbidity predict death among COVID-19 patients: results of the SARS-RAS study of the Italian society of hypertension. Hypertension 76:366-372. https:// doi.org/10.1161/HYPERTENSIONAHA.120.15324

4. Rodilla $E$, Saura A, Jiménez I, Mendizábal A, Pineda-Cantero A, Lorenzo-Hernández $\mathrm{E}$ et al (2020) Association of hypertension with allcause mortality among hospitalized patients with COVID-19. J Clin Med. https://doi.org/10.3390/ jcm9103136

5. Mancia G, Rea F, Ludergnani M, Apolone G, Corrao G (2020) Renin-angiotensin-aldosterone system blockers and the risk of Covid-19. N Eng J Med 382:2431-2440. https://doi.org/10.1056/ NEJMoa2006923

6. Reynolds HR, Adhikari S, Pulgarin C, Troxel AB, Iturrate E, Johnson SB et al (2020) Reninangiotensin-aldosterone system inhibitors and risk of Covid-19. N Engl J Med 382:2441-2448. https://doi.org/10.1056/NEJMoa2008975

7. Chen C-L, Liu L, Huang J-Y, Yu Y-L, Lo K, Huang Y-Q, Feng Y-Q (2020) Systolic blood pressure, cardiovascular mortality, and allcause mortality in normoglycemia, prediabetes, and diabetes. Diabetes Metab Syndr Obes 13:2375-2388. https://doi.org/10.2147/DMSO. S260990

8. Melgarejo JD, Yang W-Y, Thijs L, Li Y, Asayama K, Hansen TW et al (2021) Association of fatal and nonfatal cardiovascular outcomes with 24-hour 
mean arterial pressure. Hypertension 77:39-48. https://doi.org/10.1161/HYPERTENSIONAHA.120. 14929

9. Zhao D, Liu H, Dong P (2020) Liraglutide reduces systolic blood pressure in patients with type 2 diabetes mellitus: a meta-analysis of randomized trials. Clin Exp Hypertens 42:393-400. https://doi. org/10.1080/10641963.2019.1676771

10. Marre $M$ (2020) Importance of intensive blood pressure control in type 2 diabetes: Mechanisms, treatments and current guidelines. Diabetes Obes Metab 22(Suppl 2):33-42. https://doi.org/10. 1111/dom.13975

11. Mahfoud F, Mancia G, Schmieder R, Narkiewicz K, Ruilope L, Schlaich Metal (2020) Renal denervation in high-risk patients with hypertension. J Am Coll Cardiol 75:2879-2888. https://doi.org/10.1016/j. jacc.2020.04.036

12. Mahfoud F, Renkin J, Sievert H, Bertog S, Ewen S, Böhm M et al (2020) Alcohol-mediated renal denervation using the Peregrine system infusion catheter for treatment of hypertension. JACC Cardiovasc Interv 13:471-484. https://doi.org/10. 1016/j.jcin.2019.10.048

13. Ferreira JP, FitchettD, Ofstad AP, Kraus BJ, Wanner C, Zwiener I et al (2020) Empagliflozin for patients with presumed resistant hypertension: a post hoc analysis of the EMPA-REG OUTCOME trial. Am J Hypertens. https://doi.org/10.1093/ajh/hpaa073

14. Böhm M, Fitchett $D$, Ofstad AP, Brueckmann M, Kaspers S, George JT et al (2020) Heart failure and renal outcomes according to baseline and achieved blood pressure in patients with type 2 diabetes: results from EMPA-REG OUTCOME. J Hypertens 38:1829-1840. https://doi.org/10. 1097/HJH.0000000000002492

15. Jung $S$, Bosch A, Kannenkeril D, Karg MV Striepe K, Bramlage P et al (2020) Combination of empagliflozin and linagliptin improves blood pressure and vascular function in type 2 diabetes. Eur Heart J Cardiovasc Pharmacother 6:364-371. https://doi.org/10.1093/ehjcvp/pvz078

16. Inzucchi SE, Davies MJ, Khunti K, Trivedi $P$, George JT, Zwiener I et al (2020) Empagliflozin treatment effects across categories of baseline $\mathrm{HbA1C}$, body weight and blood pressure as an addon to metformin in patients with type 2 diabetes. Diabetes Obes Metab. https://doi.org/10.1111/ dom.14234

17. Papadopoulou E, Loutradis C, Tzatzagou G, Kotsa K Zografou I, Minopoulou l et al (2020) Dapagliflozin decreases ambulatory central blood pressure and pulse wave velocity in patients with type 2 diabetes: a randomized, double-blind, placebocontrolled clinical trial. J Hypertens. https://doi. org/10.1097/HJH.0000000000002690

18. Hao Z, Sun Y, Wen Y, Cui L, Li G, Liu Y (2020) Effects and mechanisms of dapagliflozin treatment on ambulatory blood pressure in diabetic patients with hypertension. Med Sci Monit 26:e925987. https://doi.org/10.12659/MSM.925987

19. Gilbert RE, Thorpe KE (2019) Acute kidney injury with sodium-glucose co-transporter-2 inhibitors: a meta-analysis of cardiovascular outcome trials. Diabetes Obes Metab. https://doi.org/10.1111/ dom.13754

20. Neuen BL, Young T, Heerspink HJL, Neal B, Perkovic V, Billot L et al (2019) SGLT2 inhibitors for the prevention of kidney failure in patients with type 2 diabetes: a systematic review and metaanalysis. Lancet Diabetes Endocrinol. https://doi. org/10.1016/S2213-8587(19)30256-6

21. Levin A, PerkovicV, WheelerDC, HantelS, George JT, von Eynatten $\mathrm{M}$ et al (2020) Empagliflozin and cardiovascular and kidney outcomes across KDIGO risk categories: post hoc analysis of a randomized double-blind, placebo-controlled, multinational trial. Clin J Am Soc Nephrol. https://doi.org/10. 2215/CJN.14901219

22. Kadowaki T, Nangaku M, Hantel S, Okamura T, von Eynatten M, Wanner C, Koitka-Weber A (2019) Empagliflozin and kidney outcomes in Asian patients with type 2 diabetes and established cardiovascular disease: results from the EMPA-REG OUTCOME ${ }^{\circledR}$ trial. J Diabetes Investig. https://doi. org/10.1111/jdi.12971

23. Butler J, Zannad F, Fitchett D, Zinman B, KoitkaWeber A, von Eynatten Metal (2019) Empagliflozin improves kidney outcomes in patients with or without heart failure. Circ Heart Fail. https://doi. org/10.1161/CIRCHEARTFAILURE.118.005875

24. Inzucchi SE, Fitchett D, Jurišić-Eržen D, Woo V, Han tel S, Janista C et al (2020) Are the cardiovascular and kidney benefits of empagliflozin influenced by baseline glucose-lowering therapy? Diabetes Obes Metab.https://doi.org/10.1111/dom.13938

25. Kraus BJ, Weir MR, Bakris GL, Mattheus M, Cherney DZI, Sattar N et al (2020) Characterization andimplications of the initial estimatedglomerular filtration rate "dip" upon sodium-glucose cotransporter-2 inhibition with empagliflozin in the EMPA-REG OUTCOME trial. Kidney Int. https://doi. org/10.1016/j.kint.2020.10.031

26. Verma S, Ji Q, Bhatt DL, Mazer CD, Al-Omran M, Inzucchi SE et al (2020) Association between uric acid levels and cardio-renal outcomes and death in patients with type 2 diabetes: a subanalysis of EMPA-REG OUTCOME. Diabetes Obes Metab. https://doi.org/10.1111/dom.13991

27. Perkovic V, Jardine MJ, Neal B, Bompoint S, Heerspink HJL, Charytan DM et al (2019) Canagliflozin and renal outcomes in type 2 diabetes and nephropathy. N Engl J Med. https://doi.org/10.1056/ NEJMoa1811744

28. Durkin M, Blais J (2020) Linear projection of estimated glomerular filtration rate decline with canagliflozin and implications for dialysis utilization and cost in diabetic nephropathy. Diabetes Ther. https://doi.org/10.1056/NEJMoa1611925

29. Oshima M, Neuen BL, Li J, Perkovic V, Charytan DM, de Zeeuw D et al (2020) Early change in Albuminuria with canagliflozin predicts kidney and cardiovascular outcomes: a posthoc analysis from the CREDENCE trial.J Am Soc Nephrol. https:// doi.org/10.1681/ASN.2020050723

30. Bakris G, Oshima M, Mahaffey KW, Agarwal R, Cannon CP, Capuano G et al (2020) Effects of canagliflozin in patients with baseline eGFR $\langle 30 \mathrm{ml} / \mathrm{min}$ per $1.73 \mathrm{~m} 2$ : subgroup analysis of the randomized CREDENCE trial. Clin J Am Soc Nephrol. https://doi.org/10.2215/CJN.10140620

31. Heerspink HJL, Stefánsson BV, Correa-Rotter R, Chertow GM, Greene T, Hou F-F et al (2020) Dapagliflozin in patients with chronic kidney disease. N Engl J Med. https://doi.org/10.1056/ NEJMoa2024816

32. Wheeler DC, Stefánsson BV, Jongs N, Chertow GM, Greene T, Hou FF et al (2021) Effects of dapagliflozin on major adverse kidney and cardiovascular events in patients with diabetic and nondiabetic chronic kidney disease: a prespecified analysis from the DAPA-CKD trial. Lancet Diabetes Endocrinol 9:22-31. https://doi.org/10.1016/ S2213-8587(20)30369-7

33. Groop P-H, Dandona P, Phillip M, Gillard $P$ Edelman S, Jendle J et al (2020) Effect of dapagliflozin as an adjunct to insulin over 52 weeks in individuals with type 1 diabetes: post-hoc renal analysis of the DEPICT randomised controlled trials. Lancet Diabetes Endocrinol 8:845-854. https://doi.org/10.1016/S2213-8587(20)30280-1 34. Scholtes RA, Muskiet MHA, van Baar MJB, Hesp AC, Greasley PJ, Karlsson C et al (2021) Natriuretic effect of two weeks of dapagliflozin treatment in patients with type 2 diabetes and preserved kidney function during standardized sodium intake: results of the DAPASALT trial. Diabetes Care 44:440-447. https://doi.org/10.2337/dc20-2604

35. Bakris GL, Agarwal R, Anker SD, Pitt B, Ruilope LM, Rossing P et al (2020) Effect of finerenone on chronic kidney disease outcomes in type 2 diabetes. N Engl J Med. https://doi.org/10.1056/ NEJMoa2025845 